'Paper presented at the 14th International Conference on

Cyclotrons and their Applications, Cape Town, October 8-13

\title{
A DEMONSTRATION OF BEAM INTENSITY MODULATION WITHOUT LOSS OF CHARGE
}

\author{
G.H. Mackenzie, W.R. Rawnsley and R. Lee
}

TRIUMF, 4004 Wesbrook Mall, Vancouver, B.C. Canada V6T $2 A 3$

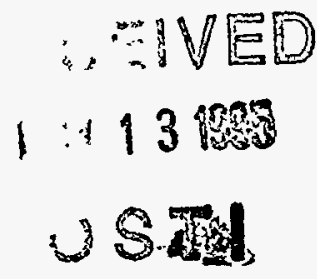

The large acceptance and the simplicity of $\mathrm{H}^{-}$extraction makes practical unusual modes of cyclotron operation. RF equipment, initially installed for $\mathrm{H}^{-}$extraction at TRIUMF, has been used to modulate the beam intensity at the extraction radius. This equipment consists of a $92 \mathrm{MHz}, 150 \mathrm{kV}$ cavity (AAC) and an RFD (11.5 MHz, $20 \mathrm{kV}$ ). The AAC augments the acceleration provided by the main $23 \mathrm{MHz}$, RF system; the RFD excites radial betatron oscillations. These devices may be operated at frequencies slightly different from their design multiple; their effect then beats with the main $\mathrm{RF}$. In this mode the AAC, for example, alternately reduces the rate of acceleration, thus increasing the overlap of turns, then enhances it, sweeping the clustered turns onto a probe or foil. Operating the AAC or RFD in this manner gathers the bulk of the charge into peaks a few microseconds wide and spaced between 6 and $50 \mu$. Changing the frequency offset alters the spacing. The peak to valley ratio was $23: 1$ and all beam was transmitted to the extraction radius.

\section{Introduction}

The programme to demonstrate the feasibility of $\mathrm{H}^{-}$extraction for the TRIUMF Kaon Factory led to the installation of two RF devices in the outer region of the cyclotron $^{1}$. These were a resonance driver, $\mathrm{RFD}$, and an auxiliary accelerating cavity, AAC. The RFD excited a radial betatron resonance, $\nu_{r}=1.5$, to generate a coherent radial oscillation. The $A A C$ has been used to double the energy gain, to flat-top the accelerating waveform, and to alter the beam microstructure ${ }^{2,3}$. This paper describes their use to alter the beam macrostructure.

\section{Elements of the Cyclotron RF System}

Fig. 1 is a plan view of the cyclotron vacuum tank showing the main accelerating system, the AAC and RFD, a $450 \mathrm{MeV}$ closed orbit and some of the extraction and

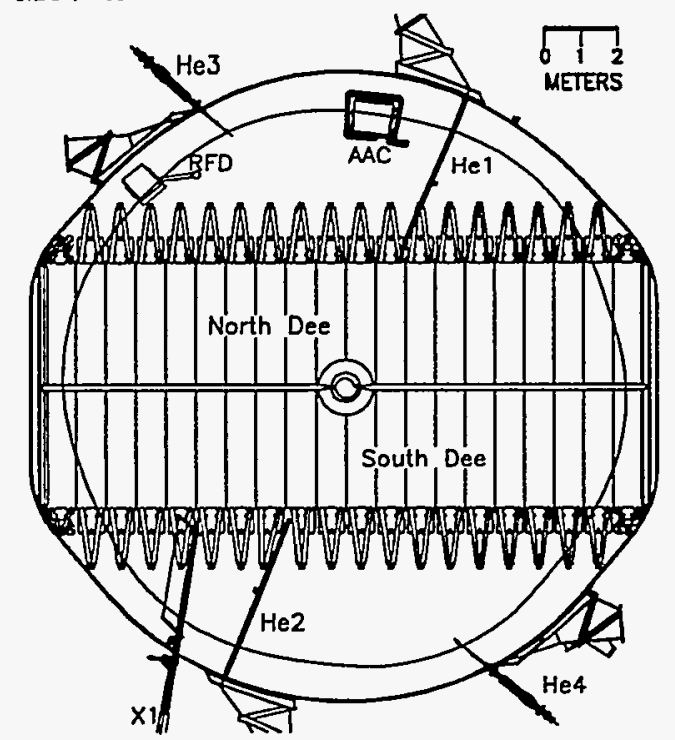

Fig. 1. A plan view of the cyclotron vacuum tank. The equipment shown is identified in the text. beam diagnostic probes (HE1-4). The $\mathrm{H}^{-}$ion orbit frequency is nominally $4.6 \mathrm{MHz}$; one particle turn taking $217 \mathrm{~ns}$. The $\lambda / 4$, dee-type, main $\mathrm{RF}$ system operates at $23 \mathrm{MHz}$, the 5 th harmonic of the orbit frequency, with a peak voltage of about $90 \mathrm{kV}$. The AAC is a $92 \mathrm{MHz}$ cavity, $\lambda / 4$ radially and $\lambda / 2$ azimuthally with a voltage peak of $140 \mathrm{kV}$, at the outer radius. The two accelerating gaps mean that the AAC can almost double or cancel the energy gain from the main RF depending on their relative phase. The RFD electrodes are at the end of a $\lambda / 4$ transmission line mounted below the vacuum tank. The tip voltage is typically $20 \mathrm{kV}$; the electrodes produce a radial electric field at $11.5 \mathrm{MHz}$ at the radius of the $\nu_{r}=1.5$ resonance which occurs at $428 \mathrm{MeV}$. During normal operation a signal picked up from the main dees is brought out of the tank and distributed to several locations. It is divided by 2 and fed to the amplifier of the RFD; multiplied by 4 and fed to the AAC preamplifier and divided by $5 \times 2^{12}$ to operate a $1.12 \mathrm{kHz}$ variable duty cycle pulser, located downstream of the ion source. For these experiments the cable from the distributed main RF signal was disconnected from the AAC and RFD amplifiers and replaced by cables from a frequency synthesizer located in the cyclotron control room.

\subsection{Application of the $A A C$}

Fig. 2 shows the radius of a particle as a function of turns made after the start of the calculation. Three situations are illustrated, (a) the AAC is not powered, (b) the AAC is on with voltage $140 \mathrm{kV}$ and with a phase to assist the energy gain per turn and (c) the AAC is powered with a phase opposing the energy gain from the main dees. If the AAC voltage were to be modulated between case (c) and (b) one might imagine a group of particles stacked in closely spaced turns in situation (c) being accelerated together rapidly outwards when the situation changes to case (b). In practice, it is easier to change the relative phase between $A A C$ and dees than vary the $A A C$ voltage. 


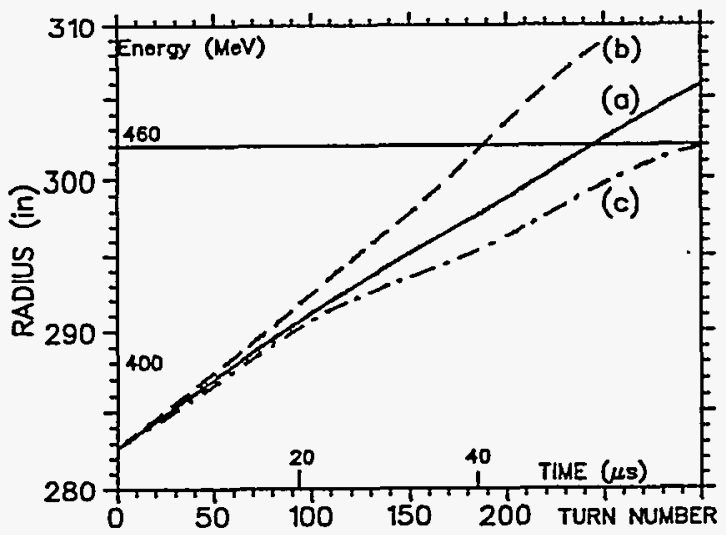

Fig. 2. Particle radius (a) AAC off (b) $A A C$ at $140 \mathrm{kV}$ assisting the dee (c) AAC opposing dee acceleration.

The frequency of beam modulation will be the beat frequency, i.e. the difference $\left(f_{A A C}-4 f_{D E E}\right)$. One would imagine that the easiest results to interpret would be those for which the beat frequency led to only one or two modulation cycles in the time taken to accelerate from, say, $400 \mathrm{MeV}$ to extraction, e.g. beat periods equivalent to 100 or 200 turns.

\subsection{Application of the RFD}

The RFD gives particles radially outward and inward kicks on successive turns in order to develop a coherent amplitude at a half integer resonance. If a particle on a given turn receives an outward kick on entering the system particles on adjacent turns, and adjacent bunches, will receive inward kicks. The two sets of particles will develop radial oscillations with the same amplitude but with different phases, Fig. 3 . Consider a particle that has an outward displacement at the point of measurement. If the voltage on the RFD is decreased the radius of the particle at that turn will decrease towards the accelerated equilibrium orbit value. The measuring device, at a fixed radius, will intercept a particle making first two turns, then four turns more. At some voltage, however, particles on that precession cycle just miss the measuring device and there is a jump to particles intercepted on

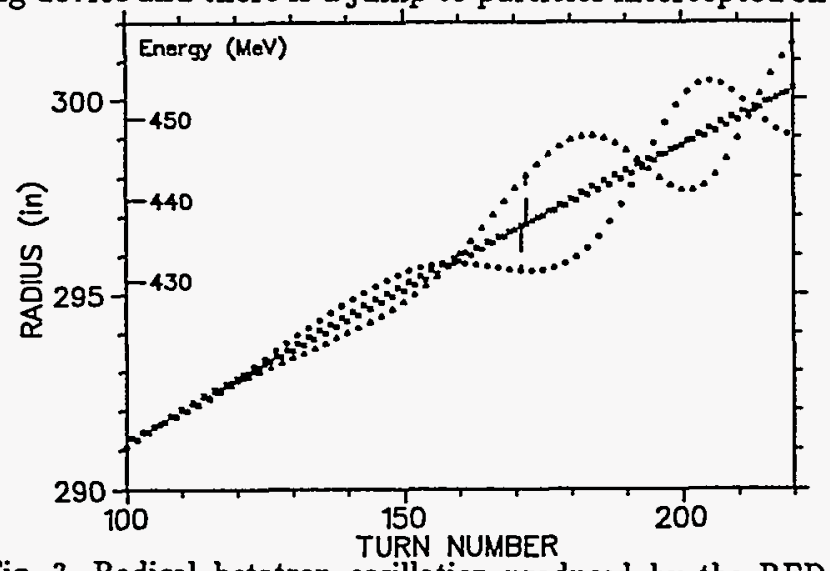

Fig. 3. Radical betatron oscillation produced by the RFD compared with a small residual oscillation (RFD off). the next precession cycle, say 10 or 20 turns later. If the RFD voltage changes sign the initial particle and turn number move further inward from the measuring device but particles in adjacent turns, and buckets, begin to move out, doubling the frequency. It would seem from the argument above, that measurements will be easiest to interpret for beat periods equivalent to a portion of a precession cycle, say 10 or 20 turns.

\section{Experiments}

The data presented here were obtained during one 6 hour period when the high extracted current beamline was inoperable. Beam in the cyclotron was stopped by a diagnostic probe at an outer radius and the probe current was amplified and displayed on an oscilloscope. The cyclotron was at its normal high current operating tune except that some flags were inserted to reduce the phase width from the usual 42 degrees ( $5 \mathrm{~ns}$ ) to about 25 degrees and the ion source pulser was run a low duty cycle. A $99.9 \%$ pulser duty factor would have delivered $70 \mu \mathrm{A}$ c.w. The pulser was not synchronized with the synthesizer signal or from a beam pick-up but with the main RF; consequently the oscilloscope, triggered by the pulser, was operated in single shot storage mode. Stable beating, or heterodyning, requires that the beat frequency be at a fixed offset from the main frequency. The synthesizer was set manually for this experiment and we relied on the dee frequency not drifting during a measurement. Asynchronous operation did not alter the beam current within the precision of measurement, $3 \%$.

\subsection{Experiments using the $A A C$}

The AAC is tuned by means of mechanical fiaps; the total frequency swing is $0.1 \%$. It was found that RF heating reduced this range to $-48 \mathrm{kHz}$ to $+32 \mathrm{kHz}$ above the nominal $4 f_{D E E}$, frequency; i.e. a beat frequency $\leq 48 \mathrm{kHz}$. The first observations were made using the signal from probe HE3 intercepting the beam at $500 \mathrm{MeV}$. The modulation period measured, $50 \mu \mathrm{s}$, agreed with that expected from the operating frequencies.

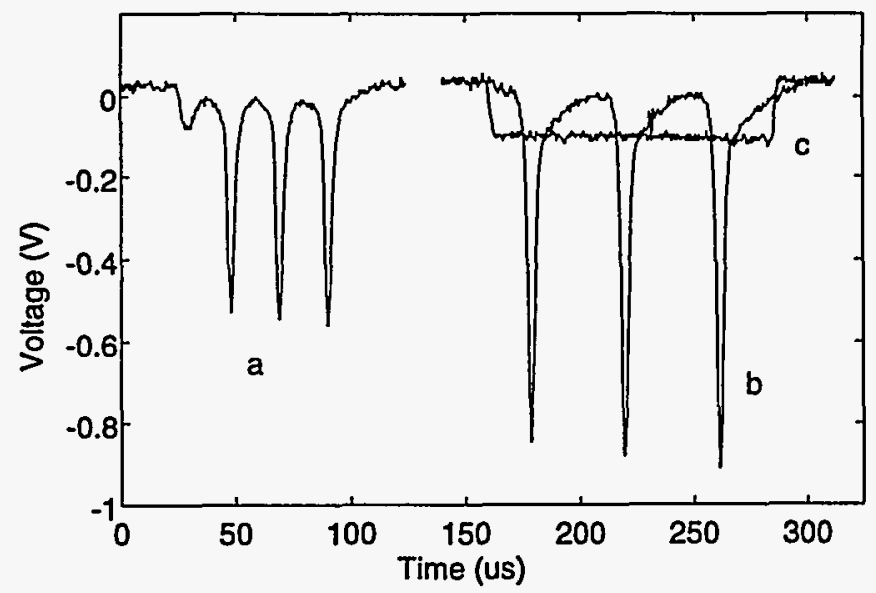

Fig. 4. Digital recording of oscilloscope trace; (a) AAC at $130 \mathrm{kV}$ and $92.2116 \mathrm{MHz}$, dee at 23.06457; (b) at $140 \mathrm{kV}$ and $92.282 \mathrm{MHz}$, dee frequency $23.06441 \mathrm{MHz}$; (c) AAC off. 


\section{DISCLAIMER}

This report was prepared as an account of work sponsored by an agency of the United States Government. Neither the United States Government nor any agency thereof, nor any of their employees, make any warranty, express or implied, or assumes any legal liability or responsibility for the accuracy, completeness, or usefulness of any information, apparatus, product, or process disclosed, or represents that its use would not infringe privately owned rights. Reference herein to any specific commercial product, process, or service by trade name, trademark, manufacturer, or otherwise does not necessarily constitute or imply its endorsement, recommendation, or favoring by the United States Government or any agency thereof. The views and opinions of authors expressed herein do not necessarily state or reflect those of the United States Government or any agency thereof. 


\section{DISCLAIMER}

Portions of this document may be illegible in electronic image products. Images are produced from the best available original document. 
Some instabilities in the ion source and bunching system had been noticed; the cyclotron was retuned and subsequent measurements were made using probe HE2 intercepting beam at $462 \mathrm{MeV}$. Beat frequencies between 21 and $50 \mathrm{kHz}$ were recorded with AAC voltages between 110 and $140 \mathrm{kV}$; examples are given in Fig. 4, a,b. The widths of the beam bunches were typically $4 \mu \mathrm{s}$ FWHM; about $60 \%$ of the beam is in the peak. In no case did the current minimum become zero. These proportions were not affected by the use of probes HE3 or HE1 to shadow HE2. The modulation pattern was stable and, apart from the beat frequency, insensitive to changes in parameters. Probe HE2 has a differential head and it would have been possible to measure separately the modulation pattern observed by the tip and body of the probe but this was not done.

\subsection{Experiments using the RFD}

The RFD could be tuned $-155 \mathrm{kHz}$ to $+15 \mathrm{kHz}$ about $f_{D E E} / 2$; i.e. true beat frequencies $\leq 155 \mathrm{kHz}$. The shape of the beam current modulation changed significantly when the RFD voltage or frequency or the probe radius were changed; see examples in Fig. 5, a,b. The modulation pattern was stable with time and over a probe radius $\pm 4 \mathrm{~mm}$. The current minima could be reduced to zero, Fig. 5d, by shadowing probe HE2 with HE1.
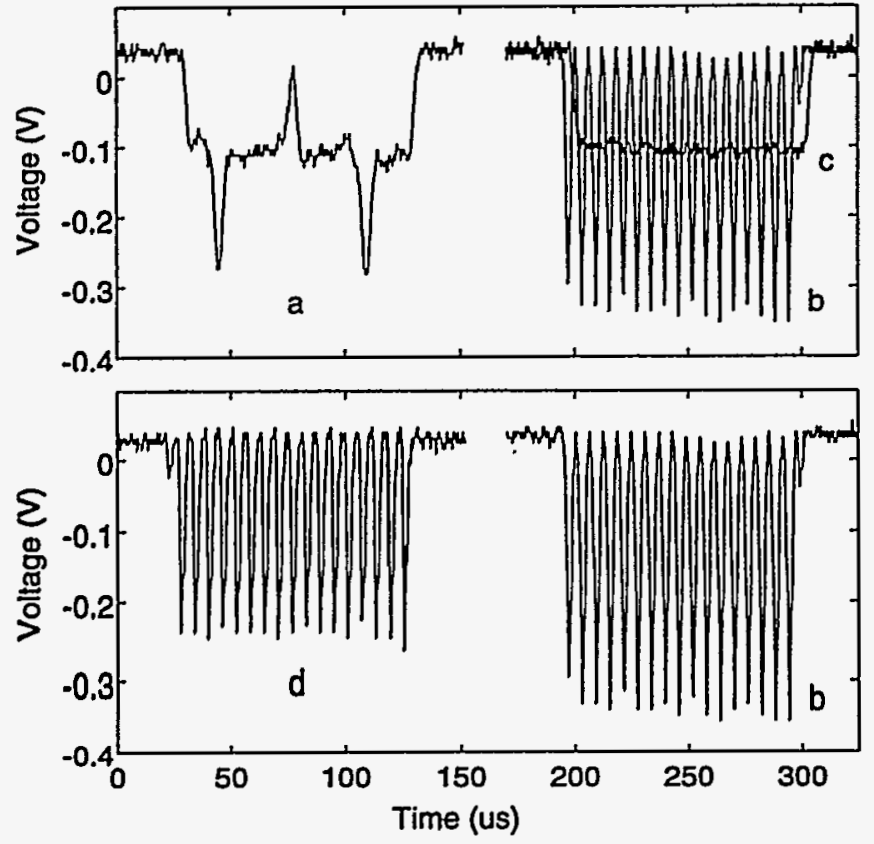

Fig, 5, RFD at (a) $11.54 \mathrm{MHz}, \mathrm{HE2}$ probe at 299.15 inch radius; (b) $11.45 \mathrm{MHz}$, probe at 301.94 inch, beat period 6.1 $\mu \mathrm{s}$; (c) RFD off, note the longer flight time to the probe; (d) as (b), but with $30 \%$ beam intercepted by HE1.

\section{Calculations and Simulations}

\subsection{General Orbit Code (GOC)}

The GOC code GOBLIN integrates numerically the equations of motion of the $\mathrm{H}^{-}$ions in the cyclotron magnetic field and in the local electric fields of the AAC and RFD. The main dee is described by a step change in energy. Single particles were tracked in these fields from 282 in. radius, $380 \mathrm{MeV}$, to 314 inch radius, $500 \mathrm{MeV}$ at the probe azimuth. The program was modified so that the first particle in a series started a "clock" which incremented by one unit for each turn of this particular particle. Other particles, with the same initial conditions, were started at regular intervals of the "clock", e.g. after the first particle had completed 30,60,90..turns. The asynchronous operation of the AAC or RFD was simulated by shifting the phase of their voltage, $V(R)$, with respect to the dee phase by a fixed amount each clock interval, e.g. the phase of the AAC would be advanced 1.957 degree for each turn of the first particle to simulate a $25 \mathrm{kHz}$ beat frequency. This phase update occurs when the particle is not in the AAC or RFD region. The radius of these particles at the probe azimuth is shown as a function of time in Fig. 6. The particles are grouped in time at the probe radius and a beam extracted by stripping would have a modulated current.

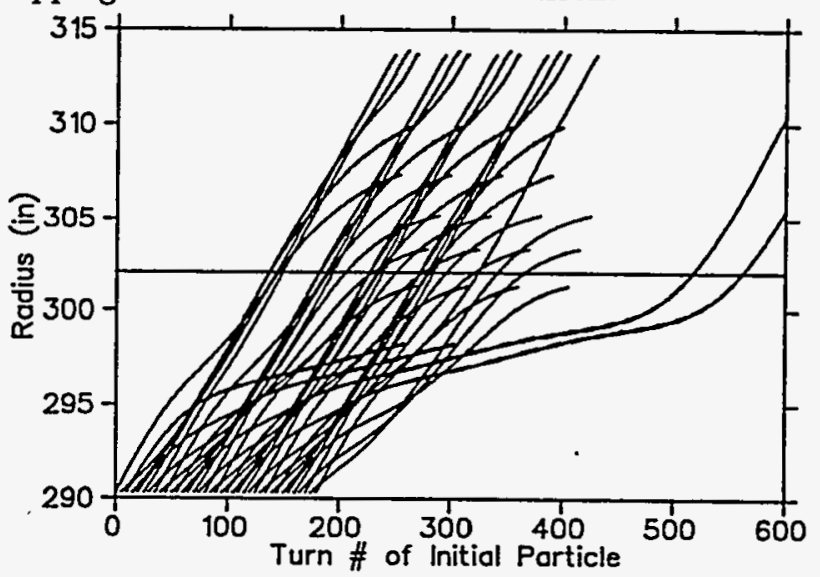

Fig. 6. Radii calculated by GOBLIN of particles started 6.5 $\mu \mathrm{s}$ apart for $\mathrm{AAC}$ asynchronous with the main dee.

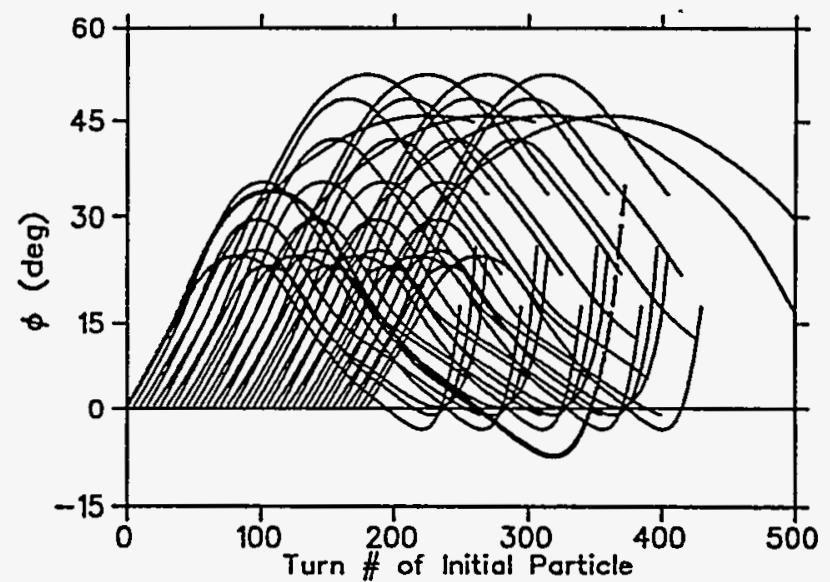

Fig. 7. Phase of particles in Fig. 9 (solid line) Phase history of the first particle with AAC off (dashed line).

Fig. 7 shows the phase with respect to the dee RF for these same particles. The normal variation due to deviations from isochronism is also shown, about \pm 20 degrees. Calculations show that the beam remains centred, i.e. $\left(x, p_{x}\right)$ small. 


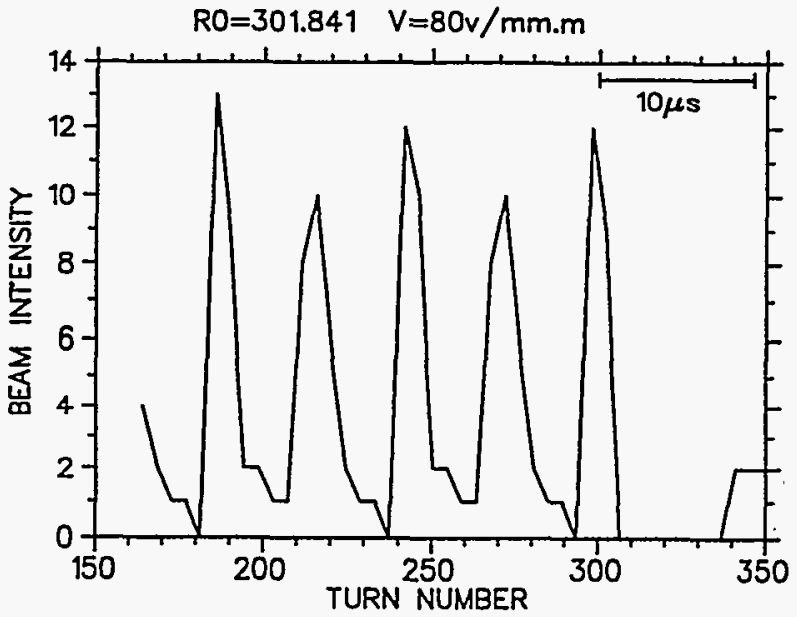

Fig. 8. Simulation of asynchronous RFD operation; the number of particles/time bin arriving at a probe radius.

The results of a similar calculation for the RFD are shown in Fig. 8, they may be compared with the experimental data in Fig. 5. It should be emphasized that the detail of the calculated current modulation is very sensitive to the parameters used for the calculation. The experiments were also sensitive to operating parameters.

\subsection{A Simple Model for the $A A C$}

Let us assume a quasi-isochronous magnetic field and let us assume that the beam is centred so that the radius as a function of energy, $\mathrm{R}(\mathrm{T})$, is known; $R \approx 10.3(\mathrm{~m}) \beta$.

Let $n$ be the turn, "clock", number and $\phi$ be the particle phase with respect to the dee and $\psi$ the particle phase with respect to the AAC and $\epsilon$ the phase advance/turn of the AAC, all in terms of the main RF $(23 \mathrm{MHz})$. Then $\mathrm{T}, \mathrm{R}, \phi$ and $\psi$ increment each turn by

$$
\Delta T=4 \hat{V}_{d e e} \cos \phi+2 \hat{V}_{A A C} \cos 4 \psi
$$

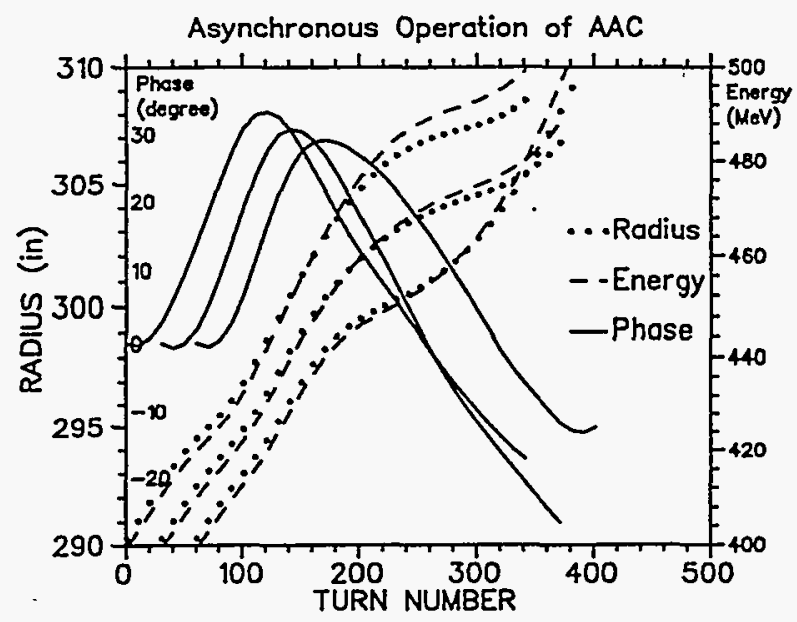

Fig. 9. Parameters calculated from Eqs. (1-3) for three particles started $6.5 \mu \mathrm{s}$ apart; $\epsilon=0.05$.

$$
\begin{gathered}
\Delta R=\left(10.3 / E_{0} \beta \gamma^{3}\right) \Delta T \\
\Delta \phi=-2 \pi h(\Delta \omega / \omega)_{i s o c h} \\
-1 / 4\left(d 2 \hat{V}_{A A C} / d T\right) \sin 4 \psi \\
\Delta \psi=\phi+\epsilon .
\end{gathered}
$$

where we assume that the dee voltage does not vary with radius and the change of $\hat{V}_{A A C}$ with time is slow.

Integration of these equations will give $R(n), T(n)$, $\phi(n)$, Fig. 9, which may be compared with Figs. 6 and 7. Particles starting at different times with respect to the AAC beat cycle will slip different amounts in regions where the field deviates from isochronism. This can sharpen the time structure. The contribution from phase compression is smaller when operating asynchronously.

\section{Conclusion}

We have shown that RF elements operated asynchronously can modulate the time structure of a cyclotron beam. Beat periods of $6 \mu \mathrm{s}$ and 25 to $50 \mu \mathrm{s}$ have been seen. The peakiness of the modulation can be affected by changes in isochronism. We have not yet extracted these beams nor measured their quality.

The idea of using the AAC or RFD to modulate the beam occurred shortly after their inception. It was decided after some computer simulations that it would be easier to explore the parameter space experimentally. It was realised that the minimum current may not be zero, nevertheless it was thought that a modulated current might increase the efficiency of $\mu \mathrm{SR}$ experiments. These experiments capture a muon and record the direction of emission of the decay electron as a function of time after capture and no new captures are desired during a measurement. It turns out that current modulation will not benefit the present experimental programme, however, it is planned to install a fast magnet to switch the muon beam between experiments after a capture. This switching would be more efficient with the time structure demonstrated. A modulated current may perturb coincidence experiments in other beamlines, but many of these are run at energies below $400 \mathrm{MeV}$ before modulation starts.

\section{Acknowledgements}

The authors would like to thank Glenn Blaker for setting up the synthesizer and determining the tuning range.

\section{References}

1. M. Zach, et al. Particle Accelerator Conf., Vancouver, IEEE NS-32, 3042 (1985).

2. R.E. Laxdal and W. Joho, Proc. European Particle Accel. Conf., Berlin, Ed. Frontières, 590 (1992).

3. R.E. Laxdal, Manipulation of beam micro- and macro-structure, this conference. 